\title{
Integrating Requirements with SLI Layer for Cloud Computing Services to Meet SLA and QoS
}

\author{
Muhammad Irfan ${ }^{1}$, Zhu Hong ${ }^{1}$, Muhammad Irfan Sharif ${ }^{2}$, Ahmed Junaid Haq Qureshi ${ }^{3}$, \\ Rakhshanda Kanwal ${ }^{4}$, Muhammad Imran ${ }^{4}$ \\ ${ }^{1}$ Collage of Computer Science and Technology, Huazhong University of Science and Technology, Wuhan, China; ${ }^{2}$ Department of \\ Computer Science COMSATS Institute of Information Technology, Wah Cantt, Pakistan; ${ }^{3}$ Anwar Chowk, Wah Cantt, Pakistan; \\ ${ }^{4}$ Courtenay Road, Woking, Surrey, England. \\ Email: scholar.mirfan@gmail.com
}

Received January $29^{\text {th }}, 2013$; revised February $27^{\text {th }}, 2013$; accepted March $9^{\text {th }}, 2013$

\begin{abstract}
Cloud computing is high technology, which fulfills needs of common as well as enterprise level to meet their information and communication technology requirements and so on. Cloud computing extends existing information technology capabilities and requirements. Many technologies are being merged with cloud computing, same as that orchestrations can boost cloud service provisioning process. The usage of orchestrations can play vital role to provision cloud services. Cloud service providers can create scalable cloud services at low cost by organizing cloud infrastructure by using cloud orchestrations. Dynamic orchestration flows can generate required cloud computing services to meet service level agreements and quality of services. There is a need to understand issues and barriers involved to integrate cloud orchestrations with cloud service provisioning process. There is also need to understand business related problems bordering cloud computing technology. There is much capacity to do targeted research work for cloud orchestrations and its integration with service level agreements as well as with SLI (service level integration) layer. In this article we have elaborated detailed analysis and identified a number of issues that will affect the cloud service users as well as cloud service providers and cloud service provisioning system. We are defining an approach to orchestrate cloud infrastructure by using orchestration flows, to generate cloud services in order to meet service level agreements and quality of standard.
\end{abstract}

Keywords: Cloud Computing; Cloud Requirements; Cloud Service Provisioning; Service Level Agreements; Quality of Service

\section{Introduction}

The development and civilization of global society, new technologies are emerging by demand of needs to fulfill requirements. Cloud computing is one of emerging technology merging all information and communication technologies, other indirectly related technologies are also linking with cloud computing. The cloud infrastructure is resource pool of cloud computing services; cloud computing services can be delivered to cloud users any time anywhere at scalable level. Cloud computing [1] is a recent technology trend aiming to deliver on demand IT resources on a pay per use basis. Cloud computing intend to provide global information technology services to common users and enterprises to host their data and services over internet. Enterprises can outsource their total infrastructure any time anywhere to global data centers of cloud computing service providers. Fox et al. [2] mentioned that, cloud computing refers to both the applications delivered as services over the internet and the hardware and system software in the datacenters that provide those services.

Main layers of cloud computing are: Software as a Service (SaaS), Platform as a Service (PaaS), and Infrastructure as a Service (IaaS). SaaS (Software as a Service) are available at Salesforce.com [3] and Clarizen.com [4]. PaaS solutions and services are available at Google AppEngine [5], Microsoft Azure [6]. IaaS hardware and its services can be approach at Amazon EC2 [7] and S3 [8], and Mosso [9]. Cloud orchestrations integrated with service level integration layer can provide cloud services at scalable level on demand. Cloud orchestrate service solution provides physical or virtual resources that are appropriate the requirements of the cloud user applications in term of CPU, memory, operating system and storage. Cloud orchestrations can enable cloud services to meet QoS parameters are recognized through a Service Level Agreement (SLA) between the cloud computing service user and cloud computing service provider.

Cloud computing recourses are pooled to serve several 
clients using a multi-tenant model, with dissimilar physical and virtual resources animatedly assigned and reassigned according to cloud user demand. There is logic of location independence in that the customer generally has no control or knowledge over the exact location of the recourse pool, provided resources. Cloud computing recourse pool consists of storage, processing, memory, network bandwidth, and virtual machines, known as Data Center. Cloud computing service provider recourse pool control and optimize resource use by leveraging a metering capability at some level of abstraction appropriate to the type of service requested by cloud user, as per SLA (Service Level Agreement). There service can be related to storage, processing, bandwidth, security, hosting and all kind of information technology services. Reserve usage can be monitored, controlled, and reported providing clearness for both the cloud service provider and cloud service user. Cloud computing services users do not have information about, where their data is being processed or stored, who is accessing their information, how their data is protected, and what data has been accessed and why? Privacy and security remain the top concerns when any service is being provided over internet and remotely resourced across the world. The migration of proprietary and sensitive data over cloud across the world is concerns for any enterprise or institutions beneficiary of cloud computing services. Understanding security in an IT environment requires transparency and control, transparency proves "who has accessed systems and data, when and where" while transparency can even pay off lack of control that's allowing monitoring to display that unofficial activity is not going on. Cloud computing, in its existing formation, requires clients to interest at considerable level for transparency and control.

Cloud computing technology has main aim to providing businesses with an array of on demand computing infrastructures and services through the internet [10]. Cloud computing therefore, promises businesses significant cost savings in procuring "local” IT infrastructures and hiring/training personnel to handle and maintain them [11,12]. There are number of problems to meet cloud computing service user requirements addressed in $[12,13]$. Cloud engineering according to requirement for cloud computing applications using Agile Service Networks (ASNs) [14]. There are number of requirements for cloud computing applications, extracted from literature [11,12,15-20].

In order to meet service level agreements cloud computing platforms should be flexible to adjust to a variety of requirements. These cloud computing platforms may deliver the infrastructure as a service to cloud computing users on demand. Therefore computing infrastructures of hardware configuration, software installation and data access demands should meet with requirements accord- ing to service level agreements and quality of standard. Different cloud based-applications have different orchestration flow and composition, configuration, and deployment requirements. The different cloud based-applications such as business applications, social networks, portals and workflows work at the top layer of the cloud computing architecture. Usability and time of these applications are not predictable, same as these applications have different requirements and different service level agreements. There are privacy and security requirements, how could personal information be protected? To protect and preserve privacy in internet based computing such as web service, online storage [21].

\section{Cloud Computing Orchestrations}

The computing “Orchestration” describes the automated arrangement, coordination, and management of complex computer systems, middleware, and services. It is often discussed as having an inherent intelligence or even implicitly autonomic control, but those are largely aspirations or analogies rather than technical descriptions. In reality, orchestration is largely the effect of automation or systems deploying elements of control theory [22]. ITU-T superficially concentrates on the cloud orchestration and broker, functional domain [23] described by FG Cloud (Focus Group on Cloud) for cloud orchestration. FG Cloud focused for policy driven automation of resource creation, allocation, tearing and operational optimization.

Service broker is an organization that deals between cloud service providers and cloud users, offering services including integration, aggregation and customization with inter cloud function [24] in cloud Ecosystem. This functionality of service orchestration domain is expanded to service brokers; they are one of component of the services orchestration architecture. As much as universe is progressing toward information and communication technologies same as mobile devices, like Smartphone or tablet, make cloud users more accessible to cloud services, ratio is directly proportional. On the other hand, standardization of cloud ecosystem and architecture that completely support inter cloud operation and orchestration is still in its early stage. Same as mentioned in the following Figure 1, as FG Cloud [23,24], showing hightech cloud architecture.

Cloud service orchestration is composing of architectture, tools and processes by humans to deliver a defined service stitching of software and hardware components together to deliver a defined service connecting and automating of work flows when applicable to deliver a defined service. The main difference between a workflow automation and orchestration is that work flows are processed and completed as processes within a single domain [22]. 


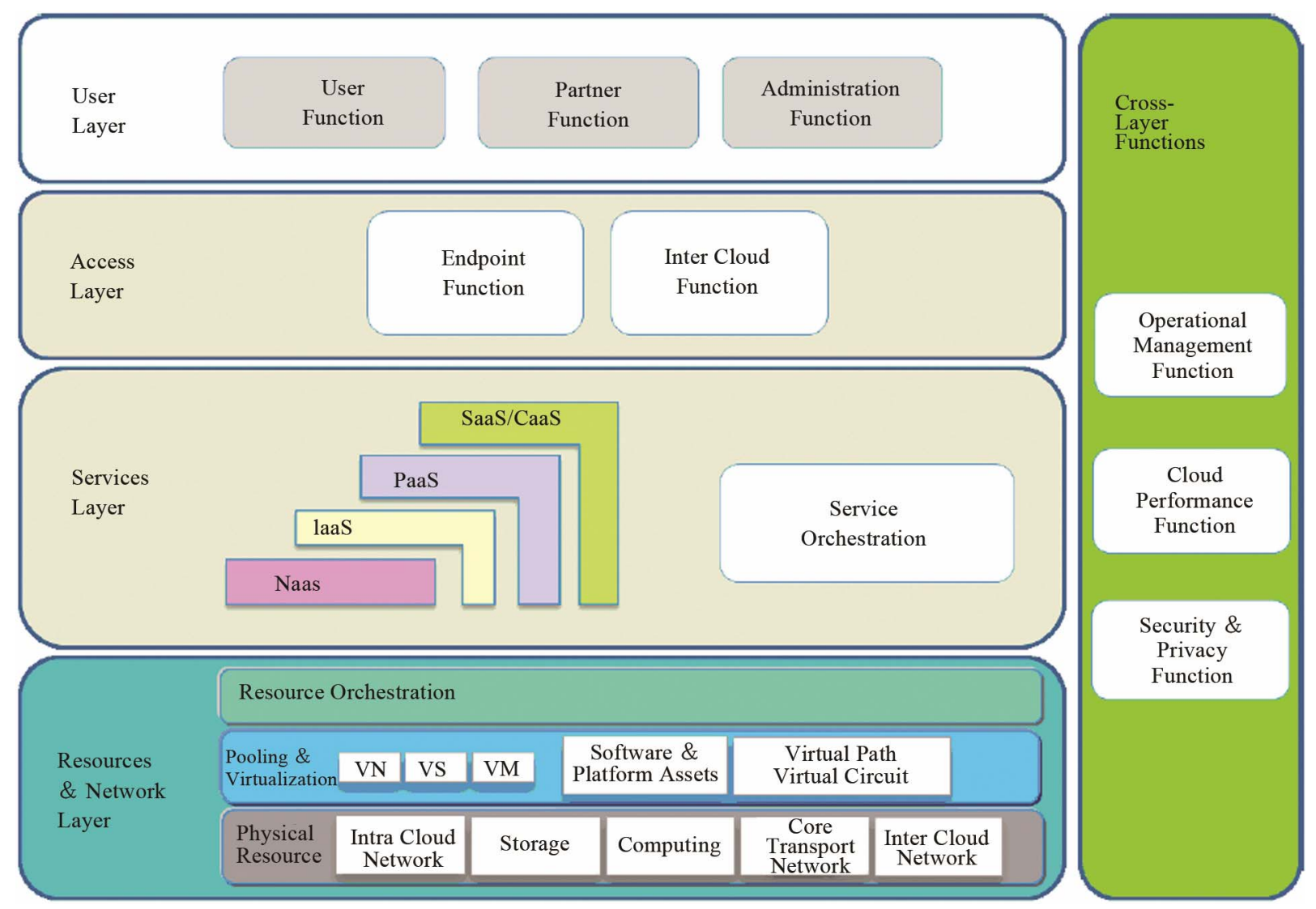

Figure 1. FG Cloud $[23,24]$ high-tech cloud architecture.

\section{Web Service Choreography and Orchestrations in Cloud Computing}

Web Service Choreography is a specification by the W3C defining a XML-based business process modeling language that describes collaboration protocols of cooperating web service participants, in which services act as peers, and interactions may be long-lived and state-full. The main effort to get choreography, The W3C Web Services Choreography Working Group, was closed on the 10th July 2009 leaving WS-CDL as a candidate recommendation [25]. Service choreography is a form of service composition in which interaction protocol between several partner services is defined from a global perspective [26]. That is, at run-time each participant in service choreography executes its part of its role according to the behavior of the other participants [27]. Choreography's role specifies the expected messaging behavior of the participants that will play it in terms of the sequencing and timing of the messages that they can consume and produce [28].

Orchestration is another term with a very similar, but still different meaning as explained in above section, orchestration is that work flows are processed and completed as processes among multiple domain [22], while workflow process with in single domain.

\section{Cloud Service Level Agreements and QoS}

Cloud service providers tries to produce maximum number of services by using less resource but still meeting service level agreements and QoS standards.

SLA defines, limits and usages and responsibilities of cloud service user and cloud service provider. SLA further gives certainty, which cloud service provider will comply with the rules about data storage; these rules are local government jurisdiction under cloud computing services. SLA also defines security requirements who and what need to control, in case of any disaster, there must be disaster recovery process defined in SLA and cloud service provider and user both agreed on this specific SLA. SLA also gives terms and conditions for cloud service provider in case if clod service is failed to keep alive. Cloud service provider must be ISO 27001 certified, in order to meet SLA and QoS standards. In 2008 Hayes pin pointed that [29] "Allowing a third party service to get custody of personal documents is awkward question about control and ownership, if you acquire cloud service can you take data with you? Could you lose access to data if you failed to pay timely?" All these issues create need and existence of service level agreements and quality of standards. SLAs can resolve and gives control over privacy and data security. 
Cloud service provider should provide cloud computing service by signing Service Level Agreements (SLAs) with customer. Cloud service user may have SLA with cloud service provider defining in SLA about memory usage and throughout in given time, CPU usage and how much bandwidth user got. Provisioning of recourses must meet SLAs, in case failed to meet SLA and QoS, cloud service provider may have penalties. Same as if cloud service user over use resource by crossing SLA, it would give loss to cloud service provider. So SLA is bond and agreement between cloud service provider and cloud service user. Still there are barriers creating conflicts to define and sign and meet SLA specifically to security and privacy. Due to unpredictable customer demand, power and software and hardware failure, conflicts are raised that might affect cloud service quality and reliability. Data ownership, data transfer, cloud service performance, reliability, security and privacy are main issues need to define clear roadmap to create trusted bond between cloud computing service provider and cloud service user. SLAs are the component empowering cloud computing to bridge this bond. Cloud computing service users demand high performance service that requires lot of resource form cloud data center resource pool. Cloud service provisioning in cloud based technology is totally depending upon Service Level Agreements (SLAs). SLA is contract between customer and cloud service provider that defines requirements of services specified as quality of service (QoS). SLA contains, functional and non functional both kind of requirements as well as defining pricing and service time line commitments, pricing and penalties. Cloud service provider ensures to meet SLA, by doing continuous monitoring of recourses and agreed terms and conditions in SLA. There are some gaps in monitoring technology, sometime monitoring is don't at higher level and lower level is ignored, resulting as breaking agreements of SLA and QoS.

An SLA is a bilateral agreement, typically between a service provider and a service consumer. These form a natural choice for representing the agreed constraints for individual jobs. While there are technologies for composing SLA (Service Level Agreement) in XML-based representations, e.g. WSLA [30], these embed domainspecific terms; no terms for resource reservation have yet been proposed within the Grid and cloud computing community. In any case, it is certain that SLA (Service Level Agreement) can be designed to include acceptable terms and conditions and standards to deliver services. For example one of SLA for any cloud computing service, start and end time bounds and a simple description of cloud resource and services requirements. SLA (Service Level Agreement) is one instrument which can be used to reserve resources in advance [31]. The time requirements and dependencies can be modeled in the SLA to guarantee the resource availability [32].

\section{Cloud Service Stacks}

Cloud computing architectures, covered following basic levels of cloud stack as defined in [33] by L. Youseff, M. Butrico and D. Da Silva Cloud computing systems fall into one following layers as shown in Figure 2, (ap- plications, software environments, software infrastruc- ture, software kernel, and hardware). At the bottom of the cloud stack is the hardware layer which is the actual physical components of the system. Some cloud computing offerings have built their system on subleasing the hardware in this layer as a service.

At the top of the stack is the cloud application layer, which is the interface of the cloud to the common computer users through web browsers and thin computing terminals.

Another cloud service stack is defined by R. W. Anderson [34] as shown in Figure 3. Anderson includes IaaS (Infrastructure as a Service) to provisioning of hardware or virtual machines on which one generally has control over the OS; therefore allowing the execution of arbitrary software. This definition isn't really enough, because there are many other kinds of infrastructure.

- Connectivity or messaging services. Examples: Microsoft BizTalk Labs and Connectivity Services, Gnip.

- Identity services. Countless OpenID identity providers, again the BizTalk Labs Identity Services.

- Data storage. Examples: Amazon's S3 and SimpleDB, Microsoft SQL Server Data Services.

There is flexible machine provisioning like Amazon EC2. These are definitely infrastructure, where the platform is the OS, Web servers, and other software. Calling this all IaaS is fine, it is all infrastructures but, maybe we should further divide these:

- Virtual Hardware Infrastructure

- Storage Infrastructure

- (Other) Infrastructure Services

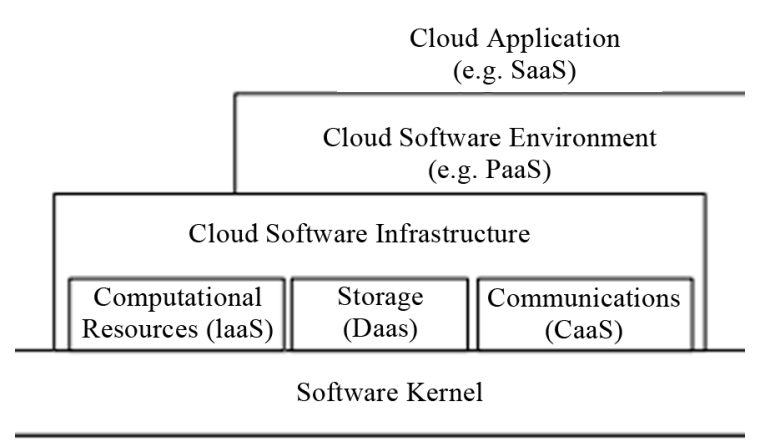

Firmware/Hardware (HaaS)

Figure 2. Cloud computing ontology [33]. 


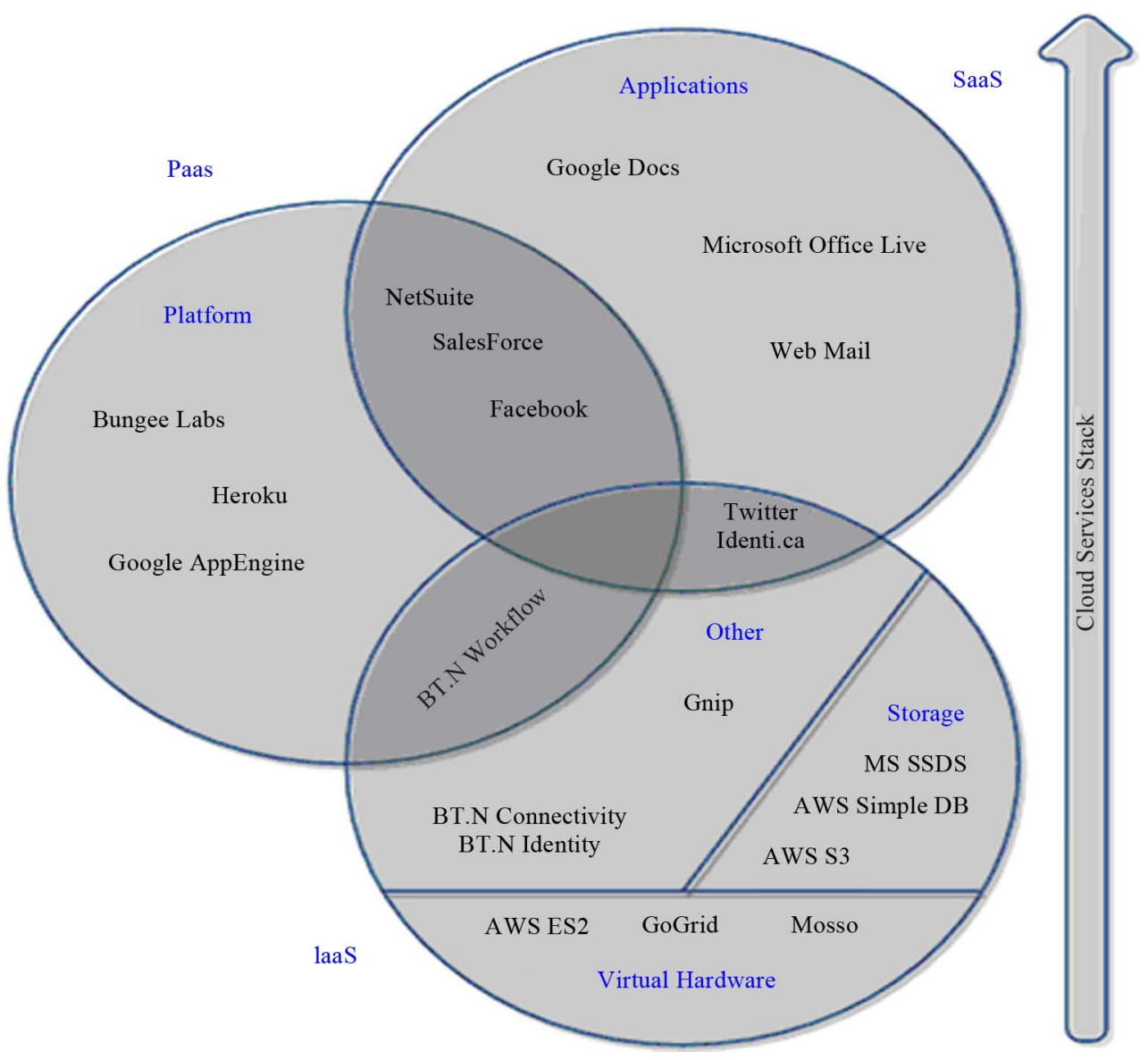

Figure 3. Cloud service stack [34].

\section{User Requirements for Cloud Computing}

Roger Clarke defines user requirements for cloud computing infrastructure [35] into main categories as listed below:

\subsection{Integrity Assurance}

Cloud computing infrastructure (CCI) must exhibit features that enable assurance of:

- Service integrity

- Data integrity

\subsection{Compliance Assurance}

CCI must exhibit features that enable users to comply with legal requirements in all jurisdictions that they are subject to, and to demonstrate that they are compliant. This encompasses:

- Service security

- Service access controls

- Data transmission security

- Data storage security

- Data use (by the service-provider)

- Data disclosure (by the service-provider to others)
- Data storage jurisdictional location

\subsection{Declaration and Measurement}

CCI must exhibit features that enable the following to be declared and measured:

- Levels of service reliability

- Protections for service survival

- Protections for data survival

- Service and data compatibility

- Service and data flexibility

\subsection{Privacy Policy Enforcement}

CCI must exhibit features that enable:

- Server privacy policies to be declared

- User privacy requirements to be declare

- Server privacy policies and user privacy requirements to be compared

- Cloud usage to be precluded where the requirements are not satisfied

\section{Service Level Tasks for SLI Layer}

There is need of such a bridge or interaction point as SLI layer which can orchestrate service level tasks to provi- 
sion required cloud computing service. For example, cloud user order for a server from cloud service provider, SLA is signed between cloud service provider and cloud service user. Cloud service provider needs to provision requested server to meet SLA and QoS. Service level layer is middle agent that mobilizes cloud computing infrastructure and initiate orchestration process, to provision and deploy server. Followings tasks as shown in Table 1, interacting with SLI layer to generate required service according to customer demand.

In Table 1 these service level tasks will be orchestrated with SLI layer, and SLI layer will run these tasks in such a way or flow provisioning required service meeting SLA and QoS.

\section{Key Value of SLI Layer}

There is need of such a bridge or interaction point as SLI layer, that is responsible for arbitrate and bridge between cloud service user and cloud computing infrastructure. More over lining of orchestrations of orchestration engine can boost cloud service provision process. The SLI layer orchestrates service tasks across cloud computing infrastructure, to provision required cloud service according to SLA and QoS. SLI layer interacts with cloud computing recourse pool to deploy service meeting SLA and QoS requirements such as response time. This specified designed SLI layer is unified central engine that is derived by service level agreements and meeting quality of standard.

\section{Conclusion}

We have elaborated service level task orchestrated with

Table 1. SLI task level table.

\begin{tabular}{cc}
\hline SLI task & Service level integration task description \\
\hline 1 & Retrieval of server order from customer \\
2 & Collection of server parameters \\
3 & Selection of server from recourse pool. \\
4 & Allocation confirmation of hardware \\
5 & Collection of list of attributes to assign \\
6 & Start hardware assignment \\
7 & Internet protocol allocation \\
8 & Operation system selection \\
9 & OS licensing \\
10 & LAN assignment \\
11 & Security rule configuration \\
12 & Internal test of server \\
13 & Delivered to related customer \\
\hline
\end{tabular}

SLI layer. Cloud stack engine will run these tasks in order to provision required cloud service. Integration cloud user requirements with service level tasks is associated by middle level layer named as SLI layer, mobilizing recourses from cloud recourse pool. Finally, provisioning required service, meets SLA and QoS, in order to deliver cloud service according to customer requirements. This idea is useful to integrate requirements with SLI tasks decreasing use of cloud service provider efforts and recourses, resulting increasing return on investment. Further work is needed to do to investigate problems to implement this idea, so researchers are invited to work on our given idea by investing their mind on this useful technology.

\section{Acknowledgements}

Thanks to researchers supported us to review piece of work encouraging us to harvest our mind, resulting as generation of service level integration with cloud user requirements.

\section{REFERENCES}

[1] L. Vaquero, L. Rodero-Marino, J. Caceres and M. Lindner, "A Break in the Clouds: Towards a Cloud Definition,” Computer Communication Review, Vol. 39, 2009, pp. 137-150.

[2] M. Armbrust, A. Fox, R. Griffith, A. D. Joseph, R. Katz, A. Konwinski, G. Lee, D. Patterson, A. Rabkin, I. Stoika and M. Zaharia, “Above the Clouds: A Berkeley View of Cloud Computing, Technical Report, UC Berkeley Reliable Adaptive Distributed Systems Laboratory,” http://abovetheclouds.cs.berkeley.edu

[3] http://www.salesforce.com

[4] http://www.clarenz.com

[5] http://code.google.com/appengine/docs/whatisgoogleappe ngine.html

[6] http://www.microsoft.com/azure/

[7] http://aws.amazon.com/ec2/

[8] http://aws.amazon.com/s3/

[9] http://www.mosso.com/

[10] D. Villegas, I. Rodero, L. Fong, N. Bobroff, Y. Liu, M. Parashar and S. M. Sadjadi, "Handbook of Cloud Computing,” Springer, Berlin, 2010.

[11] L. M. V. Gonzalez, L. Rodero-Merino, J. Caceres and M. A. Lindner, "A Break in the Clouds: Towards a Cloud Definition,” Computer Communication Review, Vol. 39, No. 1, 2009, pp. 50-55.

[12] L. Bean, "Cloud Computing: Retro Revival or the New Paradigm?” Journal of Corporate Accounting \& Finance, Vol. 21, No. 5, 2010, pp. 9-14. doi:10.1002/jcaf.20605

[13] G. Wang, “Cloud Computing,” In: G. Vossen, D. Long and J. Yu, Eds., Web Information Systems Engineering, Springer, Berlin, 2009.

[14] N. Carroll, E. Whelan and I. Richardson, “Applying So- 
cial Network Analysis to Discover Service Innovation within Agile Service Networks,” Service Science, Vol. 2, No. 4, 2010, pp. 225-244. doi:10.1287/serv.2.4.225

[15] N. Kshetri, "Cloud computing in Developing Economies,” IEEE Computer, Vol. 43, No. 10, 2010, pp. 47-55. doi:10.1109/MC.2010.212

[16] H. C. Lim, S. Babu, J. S. Chase and S. S. Parekh, “Automated Control in Cloud Computing: Challenges and Opportunities," Proceedings of the 1st Workshop on Automated Control for Datacenters and Clouds, New York, 2009, pp. 13-18. doi:10.1145/1555271.1555275

[17] M. Armbrust, A. Fox, R. Griffith, A. D. Joseph, R. H. Katz, A. Konwinski, G. Lee, D. A. Patterson, A. Rabkin, I. Stoica and M. Zaharia, "A View of Cloud Computing," Communications of the ACM, Vol. 53, No. 4, 2010, pp. 50-58. doi:10.1145/1721654.1721672

[18] B. Grobauer and T. Schreck, “Towards Incident Handling in the Cloud: Challenges and Approaches," Proceedings of the ACM Workshop on Cloud Computing Security Workshop, 2010, pp. 77-86.

[19] S. Tai, J. Nimis, A. Lenk and M. Klems, "Cloud Service Engineering," Proceedings of the 32nd ACM/IEEE International Conference on Software Engineering, Vol. 2, Cape Town, 2010, pp. 475-476. doi:10.1145/1810295.1810437

[20] L. Gu and S.-C. Cheung, "Constructing and Testing Privacyaware Services in a Cloud Computing Environment: Challenges and Opportunities," Proceedings of the First Asia-Pacific Symposium on Internetware, 2009, pp. 2:12:10. doi:10.1145/1640206.1640208

[21] H. Meziane and S. Benbernou, "A Dynamic Privacy Model for Web Services," Computer, Standards and Interfaces, Vol. 32, No. 5-6, 2010, pp. 288-304. doi:10.1016/j.csi.2010.02.001

[22] T. Erl, "Service-Oriented Architecture: Concepts, Technology \& Design,” Prentice Hall, Upper Saddle River, 2005.

[23] ITU-T Focus Group on Cloud Computing, "Cloud-Reference Architecture,” 2010.

[24] ITU-T Focus Group on Cloud Computing, “Cloud-Ref- erence Architecture,” 2011.

[25] Web Services Choreography Working Group at W3.

[26] S-Cube Knowledge Model: Service Choreography.

[27] C. Peltz, "Web Services Orchestration and Choreography,” IEEE Computer, Vol. 36, No. 10, 2003, pp. 46-52. doi:10.1109/MC.2003.1236471

[28] Z. Y. Qiu, X. P. Zhao, C. Cai and H. L. Yang, "Towards the Theoretical Foundation of Choreography," World Wide Web Conference (WWW), 2007, pp. 973-982.

[29] B. Hayes, "Cloud Computing," Communications of the ACM, Vol. 51, No. 7, 2008, pp. 9-11. doi:10.1145/1364782.1364786

[30] H. Ludwig, A. Keller, A. Dan and R. King, "A Service Level Agreement Language for Dynamic Electronic Services," Proceedings of the 4th IEEE International Workshop on Advanced Issues of E-Commerce and Web-Based Information Systems, Los Alamitos, 2002, pp. 25-32.

[31] H. Ludwig, T. Nakata, O. W. Aldrich, P. H. Wieder and W. Ziegler, "Reliable Orchestration of Resources Using WS-Agreement," Proceedings of International Conference on High Performance Computing and Communications, Berlin, 13-15 September 2006, pp. 753-762.

[32] J. MacLaren, R. Sakellariou, K. T. Krishnakumar, J. Garibaldi and D. Ouelhadj, "Towards Service Level Agreement Based Scheduling on the Grid," Workshop on Planning and Scheduling for Web and Grid Services, 2004.

[33] L. Youseff, M. Butrico and D. Da Silva, "Toward a Unified Ontology of Cloud Computing," Proceedings of Grid Computing Environments Workshop, Austin, 12-16 November 2008, pp. 1-10.

[34] R. W. Anderson, "The Cloud Services Stack-Infrastructure," 2008. http://rwandering.net/2008/07/28/the-cloud-services-stack -infrastructure/

[35] R. Clarke, "User Requirements for Cloud Computing Architecture,” 2009. http://www.rogerclarke.com/II/CCSA-0911.html 